\title{
ADVANCED DISTRIBUTED LEARNING AND COMMUNITY
}

\author{
Ulrich GYSEL and Jeffrey A. KRINOCK
}

For thousands of years, learning and teaching always took place in close proximity, and this has become firmly anchored in human consciousness.

- Otto Peters ${ }^{1}$

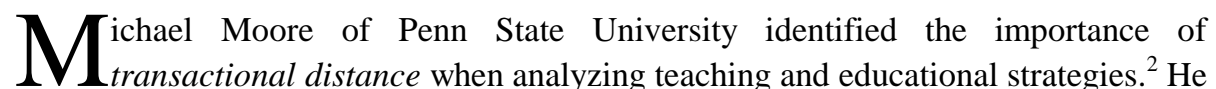
described transactional distance as [roughly] being inversely related to the extent to which teachers and students can interact during and through learning processes. Several studies and reports suggest that the educational effectiveness of the type of learning process that involves the least transactional distance-face-to-face human tutoring - is unmatched by any other form of teaching. Interestingly, many of today's proponents of advanced distributed learning emphasize mimicking the effectiveness of one-on-one human tutoring .

In terms of improving learning outcomes for individual students, this emphasis may make sense. As the work of Moore, Otto Peters, and others collectively suggests, learning techniques involving minimal transactional distance seem to be generally more effective. Accordingly, learning technologies that circumvent the detrimental effects of transactional distance may indeed result in improved short-term learning results.

This possibility has been explored in the Advanced Distributed Learning (ADL) initiative's Sharable Content Object Reference Model (SCORM). The SCORM Overview, for instance, has-in multiple versions of the SCORM-suggested Intelligent Tutoring Systems (ITS) as a technology-based means for enabling mixed initiative dialogue, allowing free form discussion between the technology and the 
student or user, a development that would effectively decrease transactional distance without directly involving a human teacher or instructor. ${ }^{3}$

In the near future we may indeed find students interacting with ITSs (or similar eLearning technologies) in a manner akin to that found today in face-to-face human tutoring. Early studies of the effectiveness of ITSs suggest we can expect, if not the same learning outcomes as those found today in one-on-one human tutoring, at least "good" learning results-relative to the learning outcomes of other e-Learning technologies.

Typically, though, the studies analyzing the learning outcomes of various e-Learning strategies focus on individual learning outcomes. The SCORM itself-arguably the worldwide technical model for assembling and distributing e-Learning-offers extensive support for tracking and reporting on individual achievements in a given course of instruction, but currently contains nothing whatsoever in the way of tracking or reporting features for the learning outcomes of groups or communities.

This paper assumes that cultures and communities have always supplied essential aspects of the learning background and learning opportunities for individuals. Accordingly, when analyzing the effectiveness of e-Learning and advanced distributed learning strategies, we should consider the impact of transactional distance (and related concepts) beyond the individual. We should regard a learning strategy's effects on the general knowledge and learning environment of cultures, subcultures and communities, not focusing on good learning outcomes for individuals alone.

\section{Effects of industrialization}

Undoubtedly the last several centuries of industrialization affected relationships between the individual and the community - to include altering the way communities and families influence (or recently, fail to influence?) the learning and training of succeeding generations. Many have researched and written about the effects of industrialization on learning and training. Several books, such as Raymond Callahan's Education and the Cult of Efficiency and John Taylor Gatto's The Underground History of American Education have analyzed how industrial concepts, expressed in such phrases as "Fordism" and "Taylorism," made their way beyond the gates of the factory and into our schools and universities. Recounting the history of the many decades of this bonding of industry and academia is beyond the scope of this article. Regarding its impact on the psyche of the average first world inhabitant, however, Langdon Winner provides a poignant analysis: 
Society is composed of persons who cannot design, build, repair, or even operate most of the devices upon which their lives depend.... In the complexity of this world people are confronted with extraordinary events and functions that are literally unintelligible to them. They are unable to give an adequate explanation of man-made phenomena in their immediate experience. They are unable to form a coherent, rational picture of the whole.... Citizens of the modern age in this respect are less fortunate than children. They never escape a fundamental bewilderment in the face of the complex world that their senses report. An objection might be raised that difficulties of the sort I have mentioned soon will have remedies. Systems theory, artificial intelligence, or some new modern way of knowing will alleviate the burdens.... Soon there will exist tools of intellectual synthesis. I must report I found no such tools in practice. I have surveyed the various candidates for this honor, systems theory and systems analysis, computer sciences and artificial intelligence, new methods of coding great masses of information, the strategy of disjointed incrementalism and so forth. As relief for the difficulties raised here none of these offers much help.... ${ }^{4}$

Umberto Eco, sounding similarly alarmed, describes an imminent media and computer-based dichotomy of society - in effect, the creation of a two-class societyas follows:

Frequently I think that our societies will be split in a short time (or they are already split) into two classes of citizens: those who only watch TV, who will receive pre-fabricated images and therefore prefabricated definitions of the world, without any power to critically choose the kind of information they receive, and those who know how to deal with the computer, who will be able to select and to elaborate information. ${ }^{5}$

Another issue-slightly different from Eco's "class" concerns-is that the direct and intentional effects of technology (e.g., the ability to create transportation independent of the need for animal power or wind, or the ability to create bombs fearsome enough to bend the will of entire nations) are only one part of the equation. The side effects of technology often seem to be just as powerful, but far less evident or understood.

Recently, into this milieu has stepped ADL and its most hopeful motto, "Anytime, anyplace." For those of us who have enjoyed the Internet's early years of unlimited and lightly restricted access to a world full of resources (ranging from the mundane to the greatest works ever compiled) it appears that the antidote to the ennui just described may be at hand. Assuming this ennui stems at least in part from our mandatory participation in systems of systems too massive to be readily 
comprehended, it is not beyond reason to hope that broad, deep, and ready access to information might provide a means for bewildered individuals to find their way out of the darkness described by Winner, Eco, and others. If families and communities have grown far too small or simple to provide understanding of, say, the risks of radon gas in the basements of homes, or the possible effects of industrial waste runoff on the family well, the Internet may indeed come to the rescue.

Many, however, have suggested (including, of course, Eco) that successful learning involves more than ready access to "empirical data" and "objective information." Hans Georg Gadamer, for example, acknowledged the role of subjectivity in even the most scientific attempts to understand the world. He emphasized linguistically encoded cultural traditions and their function in a Hegelian melding of horizons of individual understanding. In short, his philosophy taken as a whole suggests that the value of community in generating genuine learning and understanding can scarcely be overestimated. Human-to-human discourse, on a continual basis, may be an essential element of real learning, if Gadamer's understanding of how we learn is accurate.

Similarly, and more explicitly, John Taylor Gatto asserts that community and face-toface dialogue are essential to real learning. He devotes entire chapters of The Underground History of American Education to exploring what he deems to be the tragedy of a behaviorist and programmatic hijacking of the type of learning common to 1800 's America, in which education was rooted in genuine (naturally occurring?) small communities, in real, voluntary and meaningful literacy directly handed from parent (or grandparent, or neighbor) to child, of understanding handed from master to apprentice. Gatto explores the value of moral and ethical grounding, generated in close-knit families and communities, as a foundation for free individuals to launch into real literacy and genuine understanding of the world. His thesis, in part, is that community-, church-, and family-grounded upbringing very effectively prepares the individual learner for an autonomous (free?) approach to lifelong learning. To say that reducing transactional distance in learning is important to Gatto would be a significant understatement.

\section{At a crossroads: Enabling versus controlling?}

Gareth Morgan, in Images of Organization, suggests the use of metaphors to help us focus how we view organizations. A useful extract of that approach would be to employ metaphors in analyzing how we propose presenting our learning content and training within our organizations. If, for example, we deem our workers and soldiers to need training only at the point of need, and we define the point of need narrowlyfor instance, solely in terms of mission accomplishment or immediate production goals - have we perhaps leaned a bit too far in the direction of the "man as machine" and "society as system" metaphors? 
The best of advanced distributed learning concepts and technologies, employed properly, can undoubtedly provide some means to mitigate the negative effects of industrialization on our collective abilities to learn about and understand the postindustrial world, in all its complexity. That advanced distributed learning technology could likewise be used in ways that will exacerbate the problems and general ennui described by Winner, Eco, and many others, is also certainly true.

Motivations, as always, will play a central role in determining where and when ADL eases or exacerbates these problems. For instance, fiscal responsibility and efficiency-commonly advanced motivations for an ever-expanding role for advanced distributed learning - are certainly "easy sells." In the United States, the pervasiveness of defense and security concerns mandate maintenance of a vast standing army of highly trained individuals, familiar with a daunting array of technologies and fields of study. The majority of funding to keep this army highly trained, of course, comes from public coffers. (Seen from that perspective, the public education system of the US does not, perhaps, end at grade 12 (age 18) as is commonly perceived, but extends - at least for many US citizens-well into adulthood.)

If we accept that much of the DoD-provided training in the US might be viewed as a form of public education, the crucial question of motivations, the question of "controlling versus enabling," becomes even more central. In that case, H. L. Mencken's viewpoint about motivations, expressed in The American Mercury in 1924 , is perhaps worth revisiting. He suggested that neither fiscal responsibility, nor much less so, an altruistic concern for enlightened individuals, are the motivations behind any push for public education:

That erroneous assumption is to the effect that the aim of public education is to fill the young of the species with knowledge and awaken their intelligence.... Nothing could be further from the truth. The aim of public education is not to spread enlightenment at all; it is simply to reduce as many individuals as possible to the same safe level, to breed and train a standardized citizenry, to put down dissent and originality. That is its aim in the United States, whatever the pretensions of politicians, pedagogues, and other such mountebanks, and that is its aim everywhere else. ${ }^{6}$

Again, however, as a counter to Mencken's pessimism, ADL's motto of “Anytime, anyplace" offers an apparent ray of hope. Even if Mencken's pessimistic observation is correct, and the motivations behind the administration and funding of public education cannot be trusted, who is to stop the effectiveness of a Worldwide Web full 
of data, information, research, and training and educational materials from "spreading enlightenment"?

The issue of potential motivation shines through in a somewhat different light when we consider other ideas currently associated with ADL. What, for example, are the implications for popular enlightenment behind the concept of "Just-in-Time Training" (JITT)? If we explore JITT's unmistakable potential to supply procedural know-how to perhaps even illiterate workers, if we continue down that path of squeezing production from a body of workers ("personnel," "recruits," "staff") ever less aware of the "big picture," what motivations will continue to move advanced distributed learning in the direction of providing enlightenment? Will we not inevitably find access to the Internet and untold numbers of intranets more and more tightly controlled in the interest of preserving productivity?

\section{Are we willing to be superfluous? The value of autonomous learning}

Many have observed that real learning takes place when learners wish to learn. Immanuel Kant took this idea perhaps to its apex, when he said, "Those who educate use actions whose aim is to be no longer necessary."7 Kant's maxim suggests important questions about JITT and any other advanced distributed learning technology that emphasizes performance within isolated portions of processes without granting mental access and understanding to those processes as a whole. Are learners who are dependant upon task instructions provided at the last minute, devoid of all but the absolute essential details about a larger process, actually learning? And what are the broader messages that accompany a hypothetical system-wide dependence upon JITT? That Just-in-Time-Training has a place in the world is understandable; if we think of clearly written and illustrated instructions on a fire extinguisher as a form of JITT, its value in selected situations is easily evident.

What happens, however, if we are careless or excessive in our application of JITT and related technologies? What about system-wide dependence upon JITT to, for example, "improve the bottom line" without regarding JITT's effect on communities of learners. Would such carelessness be a way of saying to soldiers and workers, intentionally or not, that they need not understand the larger picture, that their input in shaping the landscape of tomorrow is not really needed? Richard Weaver, as far back as the 1940's, warned about the effects of "enforced irresponsibility" in a typical industrial worker: "Unaccustomed to determining anything about the purpose and the relationships of his work, he cannot even think in terms large enough to embrace the total situation." 8

If advanced distributed learning is not to become the digital foundation of the poorly cloaked world of control described by Mencken, Gatto, and others, we should 
perhaps find ways to analyze needs of learners (as distinguished from over-analyzing the learners themselves), ${ }^{9}$ the type of learning approach being utilized to meet those needs, and the overall effect of teaching and instructional approaches on the communities in which learning takes place. Armed with that type of "big picture" ourselves, a valuable self-awareness about both our own motivations - and perhaps the unintended consequences of the learning strategies we employ-may emerge and guide.

\section{Otto Peters and autonomous learners}

In the course of his analysis of learning in Learning and Teaching in Distance Education, Otto Peters loosely implies that promoting and nurturing autonomous learning is a sort of mark of success among distance learning strategies. Along the way to describing autonomous learning, however, he uncovers several mild (apparent) contradictions.

He quotes Schleiermacher, for instance, as saying that what "some scientificallyminded person has worked out for himself in seclusion" is "an empty shell," but later discusses how in a post-industrial society "learning is much more determined by students themselves." 10

Likewise, he discusses books as an example of some of the oldest formally structured tools of learning-perhaps implying to some that books and texts might have a limited role in promoting autonomy - but later extols the Jewish book- and literacycentric learning culture with its focused emphasis on the ability "to manage and understand texts."

These mild contradictions bring us again to consideration of the role of families and communities, in short, learning foundations and backgrounds, in creating learners capable of graduating to forms of autonomous learning. If combining basic literacy with broad access to well-structured texts was the simple formula for learning success, bright young children could, figuratively speaking, be locked into libraries and emerge years later as $\mathrm{PhD}$ equivalents. This has never been the case, and newly available "Anytime, anyplace" digital access to books and texts via advanced distributed learning will not change this.

Returning to ADL's illuminating interest in finding a technology-based means to emulate the type of human discourse found in one-on-one tutoring, Peters' analysis of distance learning again provides an interesting insight. He mentions that early progress in reducing transactional distance in distance learning involved altering texts so that they simulated a conversation between a teacher and a student. Later, addressing this idea from a somewhat different angle while discussing the Tutor 
model of distance learning, he describes the original function of a tutor (which he notes, came from England, "the mother country of the tutorial tradition"):

A tutor was typically not someone who was responsible for teaching but a fellow attached to a university who advised students on general questions concerning their studies, integrated them into college life and provided other support. Quite often there was a personal relationship between student and tutor. Tutors were therefore not teachers but advisers, and in the most favourable cases something like an older friend. One of the original meanings of the word in Latin is in fact 'protector.'

Nowadays, the term tutor is also used to define a person who provides help with learning in the narrow sense, but in contrast to the teacher model, in which the student is kept on a reasonably tight rein, this model presupposes basically that the amounts to be learnt will be learnt independently. ${ }^{11}$

An important consideration here is that that the value of tutors may not be so much their expository abilities. An even more interesting possibility is that it may not strictly be the opportunities for discourse tutors provide that is of greatest value. What if the original meaning and role of the tutor, as being one involved in mapping out the pitfalls and opportunities in a learning environment (the "protector" aspect of tutoring) is the crux of the matter? In that case, what we may be touching upon in the well-documented success of tutoring as a learning strategy is the value and role of learning foundations and backgrounds, as expressed and fulfilled in learning communities. If tutors have been part of successful learning systems without actively engaging in the "teacher" role, if they have served to "integrate students into college life," then perhaps traditional tutors, as friends and advisors, have provided collegeaged students with an important continuation of the role of family, church, and community. Tutors may create successful learners because they continue moving the student along the path to autonomous learning, providing young students - much as their parents may have done throughout childhood-with valuable informal information about the social and community aspects of a college that help maintain the college campus as a type of successful learning community.

This could help explain why tutoring - not the provision of ever-better lecturers or finer and finer texts-repeatedly comes to the forefront when comparing effectiveness of teaching and learning strategies. It may be that tutoring contributes to the health and maintenance of community - an element of learning success too easily ignored-in ways that amplify the effectiveness of other learning and teaching elements of the college environment. 


\section{Where from here?}

Thus the shift from speculative inquiry to investigation of experience has left modern man so swamped with multiplicities that he no longer sees his way.

- Richard M. Weaver, Ideas Have Consequences ${ }^{12}$

If there is a "trap" hidden within our opportunities to integrate computers ever more deeply into our training and educational systems, the trap is probably related to the criticisms of recent years, heard in legion, about the too widely used "sage on the stage" approach to teaching in our universities and colleges. Over-reliance on exposition - the pronouncing of ideas, concepts, and facts in the hope that hearing will equate to real learning and understanding - does not really work well. Carried over into e-Learning, more cleverly delivered facts, a repackaging of details and information about the "multiplicities," Weaver laments, often does little or nothing to deepen our understanding of how to live and learn, let alone why.

Computers that regurgitate in a non-human way (the only they possibly can) prerecorded information, will be repeating a form of that mistake. In effect, they will be locking instruction once again into a form of exposition, no matter how clever the algorithms and models used to sequence that exposition. ${ }^{13}$

That computers (and more to the point, networks) can enable new types of human-tohuman discourse (chat rooms, threaded discussions, and so forth) certainly offers a ray of hope in that the human element is retained. The tutor's ability to use human understanding and adjust dialogue, perhaps even with "genuine human care for the learner" as the underlying motivation, may have the best chance to re-emerge in Computer-Based Training (CBT) and Web-Based Training (WBT) not via the intelligent tutoring systems of so much hope to ADL, but in a new form of tutoring with the human retained as the source, and the computer network as the medium.

There may be evidence within fields other than e-Learning that revaluing (retaining or restoring?) the human element reaps benefits. For example, industrial management theory has in recent years embraced situational leadership. Loosely interpreted, situational leadership acknowledges that corporate environments and the individuals that form and comprise those environments have varying levels of maturity and experience calling for different management styles and approaches in different situations. Thus summarized, the concept of situational leadership sounds nearly too obvious to be of value. But today, of course, it is of value; today's managers often find advantage in applying the concepts of situational leadership. Could this be in part because situational leadership provides the manager with flexible responses that reflect the community aspects of post-industrial corporate life, overlooked and 
forgotten because nurture and growth of individuals by the corporation is at best a distant concern, if it is a concern at all?

Situational leadership may be valuable to a manager because it supplies leadership techniques that acknowledge that humans, no matter how constrained by production processes, deadlines, and accounting, still have-just as they did in yesterday's small towns and communities - varying maturity levels, different levels of experience and, in many cases, diverse understanding of the "big picture." Those variations are rooted, as they were yesterday, in how individuals integrate and react-how they learn - as they live among their peers, colleagues, and superiors.

In years past, parents, grandparents, friends and neighbors adjusted responsesthereby adjusting their families and communities - one small change at a time because they understood how individual actions, how the moral fiber and choices of their offspring and neighbors impacted the milieu in which all lived, worked and flourished (or not). In industry today, when situational leadership is insightfully applied, similar responses are possible on the part of managers and leaders. Leaders - even mid-level managers who have enough of the "big picture" regarding their industrial environments - can make leadership choices appropriate to the maturity and experience levels of individual workers, in light of the overall goals and objectives of a corporation or enterprise.

Do we indeed stand on the threshold of being able to provide digital equivalents of tutors and mentors to workers and soldiers? As we contemplate when and where to deploy such a truly advanced type of distributed learning, we should consider the value of retaining the human element, as reflected in the flexibility of situational leadership, and any other management and leadership approach that acknowledges, even tacitly, that individual workers are always part of, influenced by, and influencing the corporate community in which they labor.

\section{Notes:}

Otto Peters, Learning and Teaching in Distance Education: Pedagogical Analyses and Interpretations in an International Perspective (Sterling, VA: Stylus Publishing, 2000), 18.

2 Peters, Learning and Teaching in Distance Education, 18.

3 Philip Dodds, et al., The Sharable Content Object Reference Model, Version 1.1, Version 1.2 and SCORM 2004, Overview sections (Advanced Distributed Learning, 2001, 2002, and 2004), <http://www.adlnet.org/index.cfm?fuseaction=scormabt> (28 April 2004). 
4 Langdon Winner, Autonomous Technology: Technics-out-of-Control (1989). Quotation adapted from John Taylor Gatto, An Underground History of American Education (New York, NY: The Oxford Village Press, 2003), 314. Robert W. Roycroft and Don E. Kash describe a similar phenomenon in their The Complexity Challenge: Technological Innovation in the 21st Century (London: Pinter, 1999).

5 Umberto Eco, From Internet to Gutenberg, Part IV, Lecture at the Italian Academy for Advanced Studies in America (November 1996), <http://www.hf.ntnu.no/anv/Finnbo/ tekster/Eco/internt4.htm> (28 April 2004).

6 As quoted in Gatto, The Underground History of American Education, 1.

7 As quoted in Peters, Learning and Teaching in Distance Education, 47.

8 Richard M. Weaver, Ideas Have Consequences (Chicago: University of Chicago Press, 1948), 66.

9 Gatto describes the Chinese Dangan, a lifelong learning record, as an extreme method for cataloguing, for purposes of controlling, learners. Some see the mis-application of ADL as leading to the same end: control thinly cloaked beneath a veil of standardized opportunity.

10 Peters, Learning and Teaching in Distance Education, 35, 36.

11 Peters, Learning and Teaching in Distance Education, 25, emphasis added.

12 Weaver, Ideas Have Consequences, 13.

13 Gaming and simulations are, of course, another matter, introducing uses of computers that ignore exposition altogether. Comments about exposition and e-Learning reflect the sad reality of much of the CBT and WBT available in recent years - that it is too often a high-tech recycling of lectures, a repackaging of exposition, that was not all that effective originally, and only marginally improved, perhaps, by conversion to a computer-based delivery.

ULRICH EMANUEL GYSEL is Attaché for Information Technology at the Embassy of Switzerland in Washington DC and is currently the Co-Chair of the Partnership for Peace Consortium (PfP-C) Advanced Distributed Learning Working Group. He is also employed by the Swiss Federal Institute of Technology in Zurich as a project manager. He earned a lic.phil. in history from the University of Bern and a MS in Computer Science from the University of Wales, Cardiff, and is a certified business data processing specialist. Prior to joining the Swiss Federal Institute of Technology in Zurich in 1997, he worked for the Swiss Federal Office of Statistics and in the Swiss telecom and pharmaceutical industries.

Mr. Gysel has developed planning tools for large-scale projects, database modeling, knowledge representation, and knowledge management. Since 1999 his work has included various aspects of e-learning, to include coordination of the PfP's development of an open source Learning Management System, in addition to his leadership and participation in various joint technical and political missions employing e-learning technology in Central and Eastern Europe. He continues to actively lead, research, and publish in the fields of e-learning and knowledge management. E-mail: Ulrich.Gysel@was.rep.admin.ch.

JEFF KRINOCK, see page 80. 